\title{
Morphological variation of the relictual alveolar structures in the mandibles of baleen whales
}

\author{
Carlos M Peredo ${ }^{\text {Corresp., 1, 2, } 3}$, Nicholas Pyenson ${ }^{1,4}$ \\ ${ }^{1}$ Paleobiology, National Museum of Natural History, Smithsonian Institution, Washington D.C., District of Columbia, United States \\ 2 Earth and Environmental Science, University of Michigan - Ann Arbor, Ann Arbor, Michigan, United States \\ 3 Marine Biology, Texas A\&M University - Galveston, Galveston, Texas, United States \\ 4 Paleontology and Geology, Burke Museum of Natural History and Culture, Seattle, Washington, United States \\ Corresponding Author: Carlos M Peredo \\ Email address: cmperedo@umich.edu
}

Living baleen whales (mysticetes) are bulk filter feeders that use keratinous baleen plates to filter food from prey laden water. Extant mysticetes are born entirely edentulous, though they possess tooth buds early in ontogeny, a trait inherited from toothed ancestors. The mandibles of extant baleen whales have neither teeth nor baleen; teeth are resorbed in utero and baleen grows only on the palate. The mandibles of extant baleen whales also preserve a series of foramina and associated sulci that collectively form an elongated trough, called the alveolar groove. Despite this name, it remains unclear if the alveolar groove of edentulous mysticetes and the dental structures of toothed mammals are homologous. Here, we describe and quantify the anatomical diversity of these structures across extant mysticetes and compare their variable morphologies across living taxonomic groups (i.e., Balaenidae, Neobalaenidae, Eschrichtiidae, and Balaenopteridae). Although we found broad variability across taxonomic groups for the alveolar groove length, occupying approximately 60-80 percent of the mandible's total curvilinear length (CLL) across all taxa, the relictual alveolar foramen showed distinct patterns, ranging between $15-25 \%$ CLL in balaenids, while ranging between 3-12\% CLL in balaenopterids. This variability and the morphological patterning along the body of the mandible is consistent with the hypothesis that the foramina underlying the alveolar groove reflect relictual alveoli. These findings also lay the groundwork for future histological studies to examine the contents of these foramina and clarify their potential role in the feeding process. 
1 Morphological variation of the relictual alveolar structures in the mandibles of baleen whales

2 Carlos Mauricio Peredo $0^{1,2,3^{*}}$ and Nicholas D. Pyenson ${ }^{2,4}$

${ }^{1}$ Department of Earth and Environmental Science, University of Michigan, Ann Arbor, MI, USA

${ }^{2}$ Department of Paleobiology, National Museum of Natural History, Smithsonian Institution, Washington

5 D. C., USA

6 ${ }^{3}$ Department of Marine Biology, Texas A\&M University Galveston Campus, Galveston, TX, USA

$7 \quad{ }^{4}$ Department of Paleontology and Geology, Burke Museum of Natural History and Culture, Seattle, WA, 8 USA

*Corresponding Author: cmperedo@umich.edu 
Living baleen whales (mysticetes) are bulk filter feeders that use keratinous baleen plates to filter food from prey laden water. Extant mysticetes are born entirely edentulous, though they possess tooth buds early in ontogeny, a trait inherited from toothed ancestors. The mandibles of extant baleen whales have neither teeth nor baleen; teeth are resorbed in utero and baleen grows only on the palate. The mandibles of extant baleen whales also preserve a series of foramina and associated sulci that collectively form an elongated trough, called the alveolar groove. Despite this name, it remains unclear if the alveolar groove of edentulous mysticetes and the dental structures of toothed mammals are homologous. Here, we describe and quantify the anatomical diversity of these structures across extant mysticetes and compare their variable morphologies across living taxonomic groups (i.e., Balaenidae,

21 Neobalaenidae, Eschrichtiidae, and Balaenopteridae). Although we found broad variability across

22 taxonomic groups for the alveolar groove length, occupying approximately $60-80$ percent of the mandible's total curvilinear length (CLL) across all taxa, the relictual alveolar foramen showed distinct patterns, ranging between $15-25 \%$ CLL in balaenids, while ranging between $3-12 \%$ CLL in balaenopterids. This variability and the morphological patterning along the body of the mandible is consistent with the hypothesis that the foramina underlying the alveolar groove reflect relictual alveoli.

27 These findings also lay the groundwork for future histological studies to examine the contents of these

28 foramina and clarify their potential role in the feeding process. 


\section{Introduction}

The diversity and ecological success of baleen whales (mysticetes) has been linked to dramatic evolutionary transformations in their feeding mechanisms (Pyenson 2017; Slater et al. 2017). Living baleen whales depart from the macroraptorial feeding of their toothed ancestors and instead bulk filter feed using keratinous baleen plates (Marshall \& Goldbogen 2015; Marshall \& Pyenson 2019). Baleen whales are born entirely edentulous, although fossil relatives have mineralized teeth as adults.

Embryological evidence demonstrates extant mysticetes briefly develop teeth in utero before resorbing them prior to birth (Lanzetti 2019; Lanzetti et al. 2018; Peredo et al. 2017a).

Because teeth are resorbed in utero and baleen develops only on the palate, the body of the mandibles of extant baleen whales lack any specialized feeding structure (Peredo et al. 2017b).

Nonetheless, the mandible is still essential to the feeding process (Goldbogen et al. 2017; Pyenson et al. 2013; Shadwick et al. 2017) and at least some mysticetes have evolved novel sensory organs that facilitate feeding (Ford et al. 2013; Pyenson et al. 2012). Understanding how the edentulous mandible facilitates mysticete feeding is crucial to understanding the ecological transitions associated with the evolution of bulk filter feeding.

The mandibles of baleen whales preserve a series of dorsal foramina and associated sulci, often in such close proximity that they form a shallow groove (Figure 1). These foramina have long been considered vestigial homologues to the dental alveoli of the resorbed teeth and the shallow groove has been termed the "alveolar groove" or "alveolar gutter". Mead \& Fordyce (2009; page 42) consider "alveolar groove" to be the preferred terminology and review several other synonymous names for the structure. Recently, some authors have questioned this homology, and instead suggested that they represent distinct branches of the inferior alveolar artery or nerve and thus may have a vascular or nervous function (Peredo et al. 2017b). However, other authors have identified similar foramina in other 
edentulous mammals (Ferreira-Cardoso et al. 2019), indicating that they may indeed be homologous to alveolar structures, though leaving it uncertain if these structures are vestigial or have some function. Understanding the homology and potential function of these structures has been hampered by a lack studies detailing their morphology and variability. Here, we describe and quantify the observed morphology of these structures across the diversity of extant mysticete taxa. We report the number of foramina, the lengths of the alveolar region, and the lengths of the relictual alveolar foramen as defined by Pyenson et al. (2012) (the expanded distalmost foramen) for representative taxa of all four extant clades (including $n=34$ specimens) and compare these values across taxonomic groups. Our results provide the anatomical context necessary for subsequent histological studies to examine the contents of these foramina and thereby elucidate their potential role in the feeding process.

\section{Materials and Methods}

We examined the mandibles of 34 baleen whales deposited in the collections of the Department of Vertebrate Zoology's Division of Mammals at the Smithsonian Institution's National Museum of Natural History. Our dataset includes members from all four major taxonomic groups. For each specimen, we report six anatomical measurements from one mandible of a pair (Figure 1): the straight length of the mandible (SL); the curvilinear length of the mandible (CLL); the number of mental foramina (\#MF); the number of alveolar openings in the alveolar groove (\#AL); the length of the alveolar groove (LAG); and the length of the relictual alveolar foramen (LRAF). We also report the length of the alveolar groove and the length of the relictual alveolar foramen as percentages of the total curvilinear length (Table S1). For smaller specimens, we took measurements using an anthropometer, and for larger specimens we took measurements using a measuring tape directly on the mandibles. The measurements for straight length (SL) and curvilinear length (CLL) follow (Pyenson et al. 2013, figure 2). Additionally, we categorized each specimen based on its ontogenetic age: fetal, subadult, or adult based 


3

on suture fusion of associated cranial material (Bisconti 2001; Walsh \& Berta 2011). Specimens were designated as fetal based on collection records and as subadult based on the degree of suture fusing in the skull. The final dataset includes four fetal, three subadult, and 27 adult specimens spanning 11 total species.

\section{Results}

Our dataset comprises 34 baleen whale mandibles including 5 balaenids, 1 neobalaenid, 5 eschrichtiids, and 23 balaenopterids, 7 of which are fetal or subadult specimens. For the entire mysticete dataset, the number of mental foramina ranged from 4 (Balaenoptera acutorostrata) to 9 (Balaenoptera physalus). The number of alveolar openings in the alveolar groove ranged from 10 (Eubalaena australis and Eubalaena glacialis) to 26 (Balaenoptera physalus). Overall, both the number of mental foramina and the number of alveolar openings in the alveolar groove varied within a species and neither showed clear taxonomic patterns.

The mandibles in our dataset span the full range of mysticete body sizes, resulting in a wide variation of mandible length. The smallest adult mandible (Caperea marginata) in our dataset is $126 \mathrm{~cm}$ in curvilinear length (CLL) and the largest has a CLL of $723 \mathrm{~cm}$ (Balaenoptera musculus). Consequently, the length of the alveolar groove varies widely across out dataset as well: the minimum recorded value of the alveolar groove in an adult mandible is $86 \mathrm{~cm}$ (Eschrichtius robustus) and the maximum recorded value is $513 \mathrm{~cm}$ (Balaenoptera physalus). However, this variation is relatively constrained proportional to the mandible's CLL (Figure 2). Our results demonstrate that most taxa have alveolar groove lengths that are approximately $60-80$ percent of the mandible's total CLL. This pattern was true across all four taxonomic families and in the fetal and subadult specimens as well. Only one whale in our dataset fell notably outside these values: USNM 571340 (Balaenoptera borealis) has an alveolar groove that is only 
$10341 \%$ of the mandible's CLL.This anomalous datapoint may be the result of an unobserved pathology,

104 ontogenetic variation, or linked to the unusual feeding biomechanics of sei whales (Segre et al. 2021).

105 Despite no taxonomic pattern in the proportional length of the alveolar groove, the proportional

106 length of the relictual alveolar foramen (RAF) does vary by taxonomy (Figure 2). Balaenids and the lone

107 neobalaenid in our study have an RAF that is approximately $15-25 \%$ of the total CLL. However, most

108 adult balaenopterids have an RAF that is only approximately $3-12 \%$ of the CLL. The proportional length

109 of the RAF in balaenids is on average twice as long, and in some cases as much as five times as long, as

110 the values observed in balaenopterids (Figure 2). Eschrichtiid mandibles have values roughly between

111 balaenids and balaenopterids, approximately 9-16\% of the mandible's CLL.

112 This pattern does not, however, extend to the fetal and subadult samples in our study, all of

113 which are balaenopterids. Interestingly, the subadult balaenopterids exhibit higher values for the

114 proportional length of the RAF (13-17\% of the CLL) and the fetal specimens preserve even higher values

115 still (18-28\% of the CLL). A full comparison of the allometry of these structures across whale ontogeny is

116 beyond the scope of this project. However, our data indicate that fetal balaenopterids have RAF of

117 similar proportional length to those of balaenids and suggest that the restriction of the RAF to the distal

118 tip of the mandible may occur later in ontogeny.

119 Importantly, the variation in the proportional length of the RAF is not a function of overall

120 length of the mandible. The largest (Balaenoptera musculus) and smallest (Balaenoptera acutorostrata)

121 balaenopterids in this study both preserve some of the proportionately shortest RAF (approximately 5-6

122 and 3-9\% of the CLL respectively). In contrast, Caperea marginata, which is comparable in size to

123 Balaenoptera acutorostrata, has an RAF that is nearly $20 \%$ of the CLL, and the largest balaenids (Balaena

124 mysticetus and Eubalaena glacialis) have RAF that are approximately 14 and $18 \%$ of the CLL. This

125 suggests that, although the RAF becomes proportionately shorter throughout ontogeny in

126 balaenopterids, the pattern is not being driven simply by ontogenetic growth to larger body size.

Peer) reviewing PDF | (2021:05:61530:1:0:CHECK 18 Jun 2021) 


\section{Discussion}

The high degree of variability in the number of alveolar foramina present is noteworthy given

that it remains unclear if the alveolar foramina and the alveolar groove are actually vestigial remnants of the dentition resorbed in utero. Peredo et al. (2017b) considered their homology with teeth uncertain, in part because the mandibles of baleen whales exhibit evidence of bone remodeling similar to the patterns observed during pathological tooth loss, where the alveoli are entirely resorbed, and the bony surface becomes solid. However, recent authors (Ferreira-Cardoso et al. 2019) have identified similar structures in other edentulous mammals and considered them vestigial remnants of the resorbed dentition. Our findings suggest that the morphology of the alveolar groove and the relictual alveolar

foramen are constrained by developmental pathways early in ontogeny, supporting the hypothesis that they are homologous with the resorbed dentition (see also below about anatomical patterning). If this is the case, then it is noteworthy that these foramina and internal canals are not resorbed during the bone remodeling process (Peredo et al. 2017b), and their morphological patterning remains consistent across all four extant families. The consistency of this pattern across extant mysticetes suggests that these foramina may have been co-opted to perform a novel function in specific lineages, as documented in the case of the chin sensory organ in rorquals (Pyenson et al. 2012). However, the chin sensory organ is notably absent in balaenids, suggesting that these foramina function may more simply be related to simply innervate soft connective labial eminences (see references in Peredo et al. 2018). utero, then we would predict the high degree of variability in the number of alveoli present (ranging from 10 to 24) that we observed. Unfortunately, dental counts for embryonic mysticetes are rare, 
151 datasets of early ontogenetic variability in mysticete dentition, especially across a taxonomic breadth

152 that may elucidate an evolutionary framework to test the relationship between alveoli and tooth

153 identity. Additionally, many $19^{\text {th }}$ and early $20^{\text {th }}$ century anatomists report tooth counts for foetal

154 mysticetes, though this work is scattered across many languages and difficult to verify (see Peredo et al.

155 (2017a) for a review of embryological and histological data pertaining to mysticete tooth buds).

156 Traditionally, the ramus is defined as the vertical, non-tooth bearing portion of the mandible

157 (Mead \& Fordyce 2009 and references therein). However, extant mysticete mandibles are single

158 elongated osteological elements that lack an obvious distinction between the body of the mandible and

159 the ramus. Our results demonstrate that proportional length of the alveolar groove is tightly constrained

160 around an average of $70 \%$ of the curvilinear length of the mandible. This suggests that although extant

161 mysticete mandibles have no obvious distinction between the body and the ramus, they retain the

162 distinct anatomical patterning of their terrestrial ancestors. Based on the fossil record of mysticetes, this

163 loss of a major distinction between the body and the ramus happened no later than the early Oligocene

164 (Peredo et al. 2018), after the origin of aetiocetids but prior to the lineage leading to Maiabalaena+

165 Sitsqwayk, eomysticetids, and crown mysticetes (Peredo \& Pyenson 2018).

166 The co-option of the relictual alveolar foramen for novel functions in certain extant lineages

167 (e.g., Pyenson et al. 2012) appears to be part of a longer trend in bone remodeling that has occurred in

168 stem lineages leading to crown Mysticeti (Figure 3; and see Peredo et al. 2017b). Although stem

169 mysticetes with fully mineralized adult teeth (e.g., Salishicetus, Figure 3A) display no unusual patterns in

170 this regard, the stem mysticete Maiabalaena shows extensive cortical remodeling in the dorsal margin

171 of the mandible that is homologous with the alveolar groove in extant mysticetes (Figure 3B, 3C).

172 Interestingly, the complexity of the relictual alveolar foramen morphology in Maiabalaena is a trait that

173 appears in crownward eomysticetids, such as Waharoa ruwhenua, which may have possessed

174 mineralized teeth (Boessenecker \& Fordyce 2015). The diversity of relictual alveolar foramina in these

Peer) reviewing PDF | (2021:05:61530:1:0:CHECK 18 Jun 2021) 
175 stem lineages merits deeper examination to understand the range of alveolar morphology, any

176 associated dentition, and the distribution of internal structures related to the mandibular canal and

177 mental foramina (Peredo et al. 2017b).

178 Despite no taxonomic differences in the proportional length of the alveolar region for extant

179 mysticetes, we report substantial taxonomic differences in the proportional length of the relictual

180 alveolar foramen (Figure 2). This foramen is the distalmost opening on the dorsal margin of the

181 mandible and is elongated to as much as $20 \%$ of the CLL in balaenids and neobalaenids but constrained

182 to only about 5\% of the CLL in balaenopterids. Previous authors have reported that in balaenopterids,

183 the relictual alveolar foramen is the opening through which the lunge feeding sensory organ in the chin

184 is innervated (Pyenson et al. 2012). This sensory organ facilitates lunge feeding in balaenopterids and is

185 absent in balaenids, but it remains unclear if a homologous structure is present in other mysticete

186 groups. If the relictual alveolar foramen has been coopted to innervate the chin sensory organ, then this

187 may constrain the length of the relictual alveolar foramen in balaenopterids.

188

\section{INSTITUTIONAL ABBREVIATIONS}

USNM PAL and VZ: Departments of Paleobiology and Vertebrate Zoology (Division of Mammals),

National Museum of Natural History, Smithsonian Institution, Washington DC, USA; UWBM: Burke

192

Museum of Natural History and Culture, University of Washington, Seattle, WA, USA.

193

\section{ACKNOWLEDGEMENTS}

We would like to thank D.J. Bohaska, A.J. Millhouse, D. Lunde, and J.O. Ososky for facilitating license. 
FUNDING

201

202

203

204

205

206

207

208

209

210

211

212

213

214

215

216

217

218

219

220

221

CMP and NDP were supposed by the Remington Kellogg Fund and the Basis Foundation. CMP was further supported by the National Science Foundation (NSF Award \#1906181) and the Michigan Society of Fellows. The funders had no role in study design, data collection and analysis, decision to publish, or preparation of the manuscript.

\section{GRANT DISCLOSURES}

The following grant information was disclosed by the authors: University of Michigan Society of Fellows. NSF PRFB Award \#1906181.

\section{COMPETING INTERESTS}

Nicholas D. Pyenson is an Academic Editor for PeerJ.

\section{AUTHOR CONTRIBUTIONS}

Both authors conceived and designed the experiments, performed the analyses, drafted and edited the manuscript, prepared figures, and approved the final draft. CMP measured the mysticete mandibles.

\section{DATA AVAILABILITY}

The following information was supplied regarding data availability: The full raw data file is available in the Supplemental Files.

222 
223 SUPPLEMENTAL INFORMATION

224 Supplemental information for this article can be found online at:

225

226 


\section{REFERENCES}

Bisconti M. 2001. Morphology and postnatal growth trajectory of Rorqual Petrosal. Italian Journal of Zoology 68:87-93. 10.1080/11250000109356390

Boessenecker RW, and Fordyce RE. 2015. Anatomy, feeding ecology, and ontogeny of a transitional baleen whale: a new genus and species of Eomysticetidae (Mammalia: Cetacea) from the Oligocene of New Zealand. PeerJ 3:1-69. 10.7717/peerj.1129

Ferreira-Cardoso S, Delsuc F, and Hautier L. 2019. Evolutionary Tinkering of the Mandibular Canal Linked to Convergent Regression of Teeth in Placental Mammals. Current Biology. 10.1016/j.cub.2018.12.023

Ford TJ, Jr., Werth AJ, and George JC. 2013. An intraoral thermoregulatory organ in the bowhead whale (Balaena mysticetus), the corpus cavernosum maxillaris. Anat Rec (Hoboken) 296:701-708. 10.1002/ar.22681

Goldbogen JA, Cade DE, Calambokidis J, Friedlaender AS, Potvin J, Segre PS, and Werth AJ. 2017. How baleen whales feed: the biomechanics of engulfment and filtration. Annual Review of Marine Science 9:367-386. 10.1146/annurev-marine-122414-033905

Ishikawa H, and Amasaki H. 1995. Development and physiological degradation of tooth buds and development of rudiment of baleen plate in southern minke whale, Balaenoptera acutorostrata. Journal of Veterinary Medical Science 57:665-670.

Ishikawa H, Amasaki H, Dohguchi H, Furuya A, and Suzuki K. 1999. Immunohistological distributions of fibronectin, tenascin, type I, III and IV collagens, and laminin during tooth development and degeneration in fetuses of minke whale, Balaenoptera acutorostrata. Journal of Veterinary Medical Science 61:227-232.

Lanzetti A. 2019. Prenatal developmental sequence of the skull of minke whales and its implications for the evolution of mysticetes and the teeth-to-baleen transition. J Anat 235:725-748. 10.1111/joa.13029

Lanzetti A, Berta A, and Ekdale EG. 2018. Prenatal development of the humpback whale: Growth rate, tooth loss and skull shape changes in an evolutionary framework. Anat Rec (Hoboken). 10.1002/ar.23990

Marshall CD, and Goldbogen JA. 2015. Feeding mechanisms. In: Castellini MA, and Mellish J-A, eds. Marine Mammal Physiology: Requisites for Ocean Living: CRC Press, 95-118.

Marshall CD, and Pyenson ND. 2019. Feeding in aquatic mammals: an evolutionary and functional approach. Feeding in Vertebrates: Springer, 743-785.

Mead JG, and Fordyce RE. 2009. The therian skull: a lexicon with emphasis on the odontocetes. Smithsonian Contributions to Zoology 627:1-248.

Peredo CM, and Pyenson ND. 2018. Salishicetus meadi, a new aetiocetid from the late Oligocene of Washington State and implications for feeding transitions in early mysticete evolution. Royal Society Open Science 5:172336. 10.1098/rsos.172336

Peredo CM, Pyenson ND, and Boersma AT. 2017a. Decoupling tooth loss from the evolution of baleen in whales. Frontiers in Marine Science 4:1-11. 10.3389/fmars.2017.00067

Peredo CM, Pyenson ND, Marshall CD, and Uhen MD. 2018. Tooth loss precedes the origin of baleen in whales. Current Biology 28:3992-4000. 10.1016/j.cub.2018.10.047

Peredo CM, Pyenson ND, Uhen MD, and Marshall CD. 2017b. Alveoli, teeth, and tooth loss: Understanding the homology of internal mandibular structures in mysticete cetaceans. PLoS ONE 12:1-26. 10.1371/journal.pone.0178243

Pyenson ND. 2017. The ecological rise of whales chronicled by the fossil record. Current Biology 27:R558-R564. 10.1016/j.cub.2017.05.001 
Pyenson ND, Goldbogen JA, and Shadwick RE. 2013. Mandible allometry in extant and fossil Balaenopteridae (Cetacea: Mammalia): the largest vertebrate skeletal element and its role in rorqual lunge feeding. Biological Journal of the Linnean Society 108:586-699. 10.1111/j.10958312.2012.02032.x

Pyenson ND, Goldbogen JA, Vogl AW, Szathmary G, Drake RL, and Shadwick RE. 2012. Discovery of a sensory organ that coordinates lunge feeding in rorqual whales. Nature 485:498-501. $10.1038 /$ nature11135

Segre PS, Weir CR, Stanworth A, Cartwright S, Friedlaender AS, and Goldbogen JA. 2021. Biomechanically distinct filter-feeding behaviors distinguish sei whales as a functional intermediate and ecologically flexible species. Journal of Experimental Biology 224. 10.1242/jeb.238873

Shadwick RE, Goldbogen JA, Pyenson ND, and Whale JCA. 2017. Structure and Function in the Lunge Feeding Apparatus: Mechanical Properties of the Fin Whale Mandible. The Anatomical Record 300:1953-1962. 10.1002/ar.23647

Slater GJ, Goldbogen JA, and Pyenson ND. 2017. Independent evolution of baleen whale gigantism linked to Plio-Pleistocene ocean dynamics. Proceedings of the Royal Society B: Biological Sciences 284:20170546. 10.1098/rspb.2017.0546

Thewissen JGM, Hieronymus TL, George JC, Suydam R, Stimmelmayr R, and McBurney D. 2017. Evolutionary aspects of the development of teeth and baleen in the bowhead whale. Journal of anatomy 230:549-566. 10.1111/joa.12579

Walsh BM, and Berta A. 2011. Occipital ossification of balaenopteroid mysticetes. Anat Rec (Hoboken) 294:391-398. 10.1002/ar.21340 


\section{Figure Captions}

298 Figure 1: (A) Left mandible of Balaenoptera acutorostrata (USNM VZ 571487) in medial view with

299 selected regions enhanced in panel (B). (B) Enhanced view of the selected regions on panel (A),

300 specifically highlighting the alveolar groove, associated sulci, the relictual alveolar foramen at the distal

301 terminus of the mandible, and the symphyseal groove, using CT data. (C) Left mandible of Balaenoptera

302 acutorostrata (USNM VZ 571487) in dorsal view demonstrating the curvilinear length (CLL) and the

303 straight length (SL) measured in this study. (D) Left mandible of Balaenoptera acutorostrata (USNM VZ

304571487 ) in medial view demonstrating the length of the alveolar groove (LAG) and the length of the

305 relictual alveolar foramen (LRAF) measured in this study.

306

307 Figure 2: Length of the alveolar groove (orange) and the length of the relictual alveolar foramen (blue)

308 as proportions of the total curvilinear length of the mandible. This graph includes all adult specimens in 309 our study but excludes the subadult and fetal specimens reported in Table S1.

311 Figure 3: Mandibles of a toothed stem mysticete (A) UWBM 50004: Salishicetus meadi; an edentulous

312 stem mysticete (B) USNM PAL 314627: Maiabalaena nesbittae; and an extant mysticete (C) USNM VZ

313 571847: Balaenoptera acutorostrata. Mandibles are figured in oblique view and in cross sectional slices

314 from CT scans to demonstrate the morphological variability of the distal alveolus or associated foramina. 


\section{Figure 1}

Figure 1

Figure 1: (A) Left mandible of Balaenoptera acutorostrata (USNM VZ 571487) in medial view with selected regions enhanced in panel (B). (B) Enhanced view of the selected regions on panel (A), specifically highlighting the alveolar groove, associated sulci, the relictual alveolar foramen at the distal terminus of the mandible, and the symphyseal groove, using CT data. (C) Left mandible of Balaenoptera acutorostrata (USNM VZ 571487) in dorsal view demonstrating the curvilinear length (CLL) and the straight length $(\mathrm{SL})$ measured in this study. (D) Left mandible of Balaenoptera acutorostrata (USNM VZ 571487) in medial view demonstrating the length of the alveolar groove (LAG) and the length of the relictual alveolar foramen (LRAF) measured in this study. 

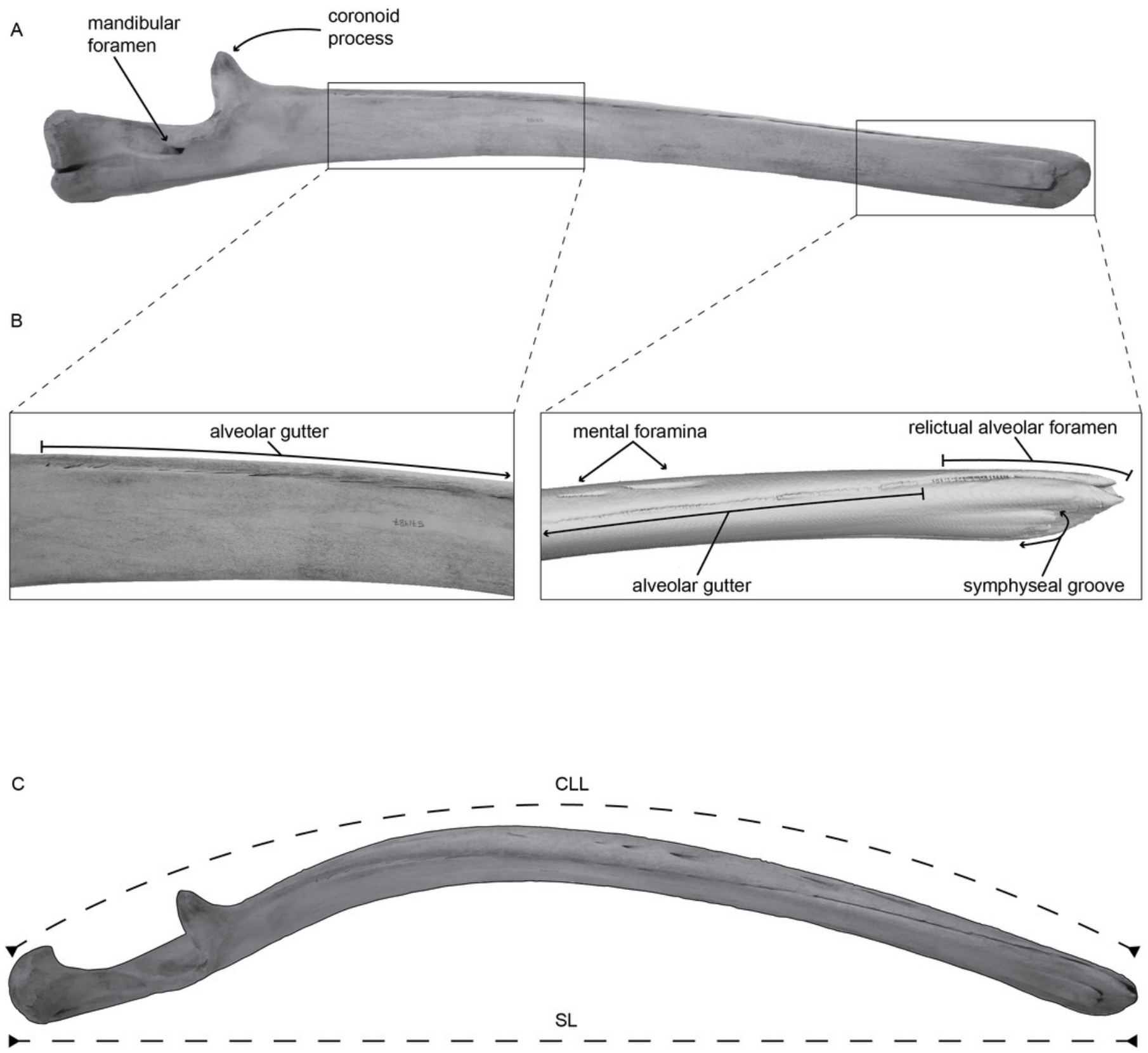

D

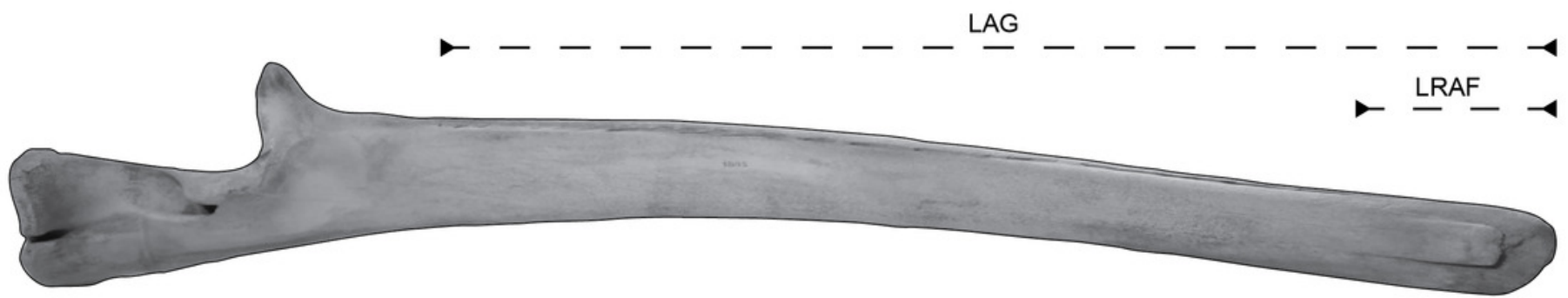


Figure 2

Figure 2

Figure 2: Length of the alveolar groove (orange) and the length of the relictual alveolar foramen (blue) as proportions of the total curvilinear length of the mandible. This graph includes all adult specimens in our study but excludes the subadult and fetal specimens reported in Table S1.

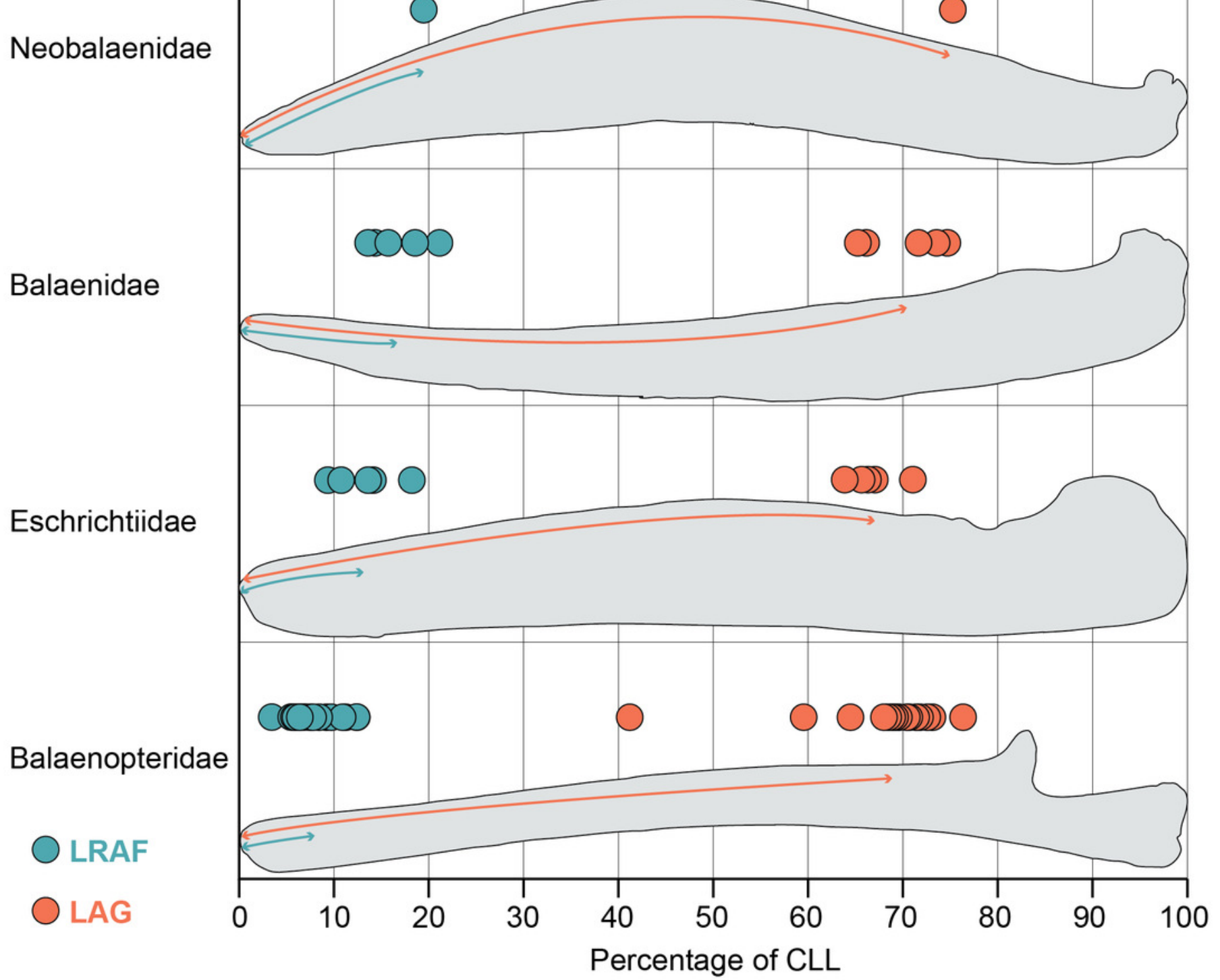




\section{Figure 3}

Figure 3

Figure 3: Mandibles of a toothed stem mysticete (A) UWBM 50004: Salishicetus meadi; an edentulous stem mysticete (B) USNM PAL 314627: Maiabalaena nesbittae; and an extant mysticete (C) USNM VZ 571847: Balaenoptera acutorostrata. Mandibles are figured in oblique view and in cross sectional slices from CT scans to demonstrate the morphological variability of the distal alveolus or associated foramina. 


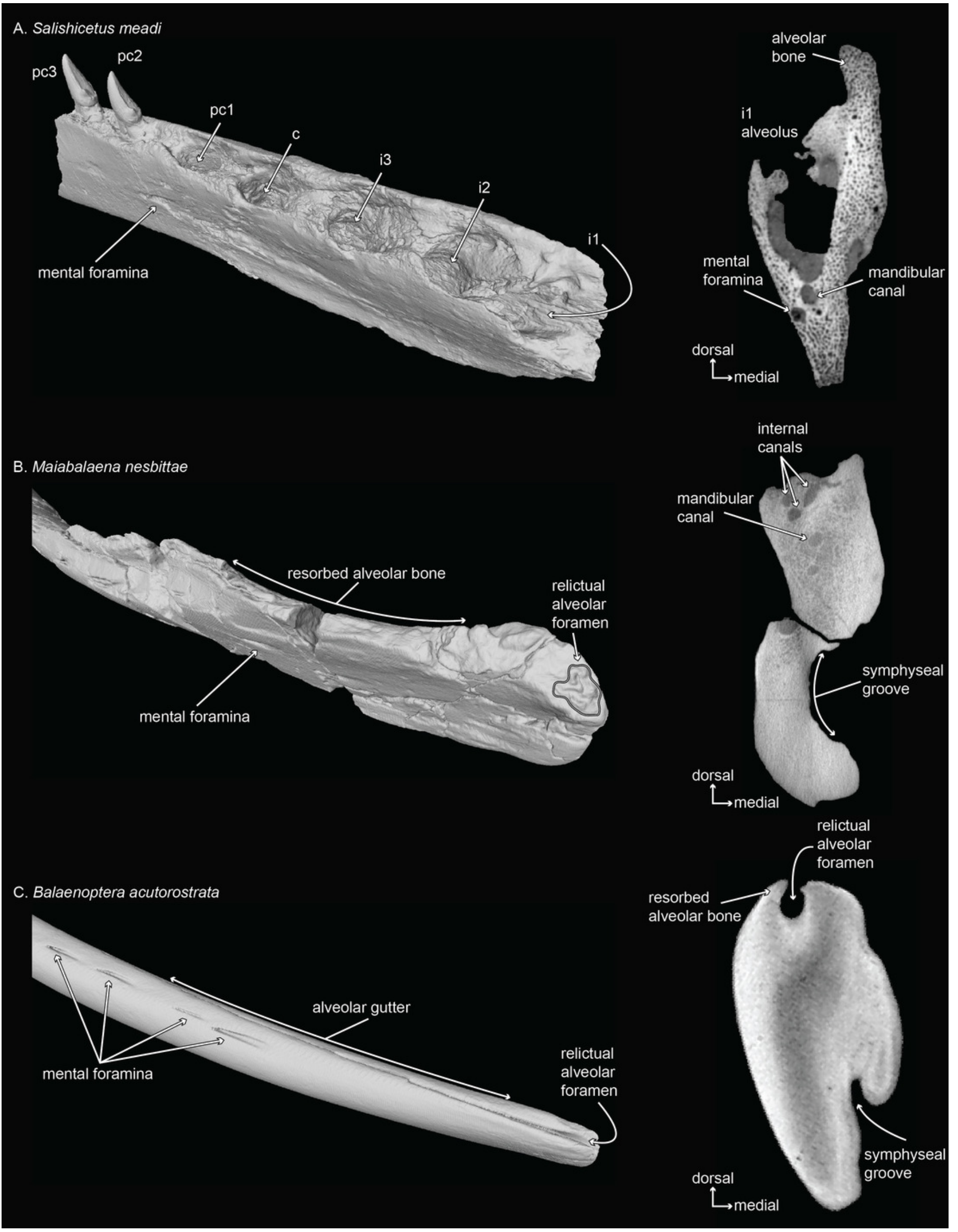

